\title{
Content Publishing and Downloading Practice in BitTorrent
}

\author{
Seungbae Kim ${ }^{1}$, Jinyoung $\mathrm{Han}^{2}$, Taejoong Chung ${ }^{2}$, Hyun-chul Kim³ \\ Ted "Taekyoung" Kwon" ${ }^{2}$, and Yanghee Choi ${ }^{2}$ \\ ${ }^{1}$ KAIST Institute for Information Technology Convergence, Korea \\ 2 Seoul National University, Korea \\ 3 Sangmyung University, Korea \\ sbkim@itc.kaist.ac.kr, \{jyhan,tjchung\}@mmlab.snu.ac.kr, \\ hkim@smu.ac.kr, \{tkkwon, yhchoi\}@snu.ac.kr
}

\begin{abstract}
BitTorrent has been popular over the last decade. However, few studies have made serious efforts to understand who and why publish torrents, and what strategies are adopted by publishers. In this paper, we study the current content publishing practice in BitTorrent from a socio-economic point of view, by unraveling (1) how files are published by publishers, (2) what strategies are adopted by publishers, and (3) how effective those strategies are. To this end, we conduct comprehensive measurements on one of the largest BitTorrent Portal, The Pirate Bay (TPB). From the datasets of $52 \mathrm{~K}$ torrents and $16 \mathrm{M}$ users, we classify the content publishers into three types: (i) fake publishers, (ii) profit-driven publishers, and (iii) altruistic publishers. We show that a significant amount of traffic $(61 \%)$ of BitTorrent has been generated (i.e., unnecessarily wasted) to download fake torrents. Therefore, we suggest a method to filter out fake publishers on TPB by considering their distinct publishing patterns learned from our measurement study, and show the proposed method can reduce around $45 \%$ of the total download traffic. We also reveal that profit-driven publishers adopt different publishing strategies according to their revenue models (e.g., advertising private tracker sites to attract potential new members, or exposing image URLs to make people click the URL links).
\end{abstract}

Keywords: BitTorrent, Content Publishing, Measurement.

\section{Introduction}

BitTorrent [1] is one of the most popular systems for sharing contents. According to the Ipoque's report released in 2009 [2], BitTorrent accounts for approximately $27-55 \%$ of the Internet traffic. The huge success of BitTorrent is due to some attractive properties of its ecosystem. First, its swarming mechanism achieves good performance in terms of throughput and scales well even in the presence of massive flash crowds for popular contents. Second, popular (and often copyrighted) torrents (e.g., Hollywood movies) are available to users with no cost. 
This phenomenal success has attracted the research community to examine the performance aspects of BitTorrent in terms of throughput, fairness and incentive issues [9 12]. However, the socio-economic aspects of BitTorrent have received relatively little attention. In particular, to our knowledge, few studies have made serious efforts to investigate who and why publish contents, and what strategies are adopted by publishers.

Recently, some studies have analyzed the demography of BitTorrent publishers and showed a highly skewed distribution of the number of published files [7, 8]. Also, a significant portion of publishers belong to a small number of hosting service providers [5, 8]. Based on the above recent findings, Cuevas et al. [5] investigated whether content publishers behave in an altruistic fashion or have other incentives such as financial gains. Their study revealed that content publishing in BitTorrent is largely driven by financial incentives. More recently, Kryczka et al. [6] conducted a measurement study to analyze the fake content publishing phenomenon: (i) a few tens of publishers are responsible for $90 \%$ of the fake torrents, and (ii) more than $99 \%$ of the analyzed fake torrents are related to either malware or scam web sites. These studies help us understand who publish content in BitTorrent and for what purposes.

In this paper, we study the content publishing practice in BitTorrent from a socio-economic point of view by unraveling (1) how torrents are published by publishers of different types (i.e., fake, profit-driven, and altruistic, as detailed below.) (2) what strategies are adopted by those publishers, and (3) whether and how those strategies are effective. To this end, we conduct a comprehensive measurement on one of the largest BitTorrent portals, The Pirate Bay (TPB) [3]. We have collected the datasets that contain more than $52 \mathrm{~K}$ published torrents and $16 \mathrm{M}$ users for 38 days, from March 25 to May 1, 2011. Using the datasets, we first classify publishers into three types, as in [5]: (i) fake publishers who try to distribute fake torrents, (ii) profit-driven publishers who seek to obtain financial gains, and (iii) altruistic publishers who wish to share contents with others. Note that while Cuevas et al. [5] analyzed only top 100 publishers (in terms of the number of published torrents), we classify top 861 publishers who have uploaded al least 10 torrents. The 861 publishers are responsible for $67 \%$ of all the published torrents. We also investigate strategies adopted by publishers such as content publishing rates, content categories like movie or music, and advertising strategies of profit-driven publishers. Moreover, we show how effectively these strategies are working by looking at the popularity of contents and user dynamics in the swarms.

In our study, we notice that fake publishers are responsible for a large portion of BitTorrent traffic. We reveal that fake publishers are estimated to be responsible for around $61 \%$ of download traffic, which indicates that a significant amount of network bandwidth has been just wasted to transfer the fake torrents. Therefore, we suggest a method to detect fake publishers and show that the proposed method can significantly reduce the estimated amount of BitTorrent traffic, by as much as $45 \%$. We also reveal that profit-driven publishers adopt different strategies according to their revenue models. 
The main contributions of this paper are as follows:

- This is a comprehensive measurement study on the content publishing practice by observing one of the largest BitTorrent portals. We make our developed codes and anonymized datasets publicly available 1

- We find that profit-driven publishers among the top 100 publishers constitute the majority (around 54\%) in terms of the number of publisher's TPB accounts while the portion of altruistic and fake publishers are $29 \%$ and $17 \%$, respectively, which is in line with the study in [5]. However, fake publishers among the top 101 861 publishers are dominant (around 50\%) while the portion of altruistic and profit-driven publishers are $27 \%$ and $23 \%$, respectively.

- We reveal that fake publishers' contents are responsible for around $61 \%$ of the estimated total download traffic. If those fake torrents can be effectively filtered out in advance, a significant amount of BitTorrent-caused traffic will be reduced, allowing users to avoid downloading such fake torrents.

- We suggest a method considering publishing rate (i.e., the average time interval between two consecutive publications) of publishers to effectively filter out fake publishers. We show that this simple solution can save $45 \%$ of the total download traffic, caused by those fake torrents.

- There are primarily two kinds of profit-driven publishers: i) who want to advertise private trackers $(51 \%$ and $67 \%$ of the total number of profit-driven publishers and the number of published torrents, respectively) by uploading TV contents $(53 \%)$, and ii) who want to advertise image hosting sites $(26 \%$ and $21 \%$ of the total number of profit-driven publishers and the number of published torrents, respectively) by uploading Porn contents (82\%).

The rest of the paper is organized as follows. Section 2 describes our measurement methodology and criteria for publisher classification. In Section 3, we analyze how many torrents are published by each publisher type. We investigate publishers' strategies to achieve their goals, and how effective those strategies are in Sections 4 and 5, respectively. After discussing implications on publishers' strategies in Section 6, we conclude this paper in Section 7

\section{Methodology}

\subsection{Data Collection}

We conduct a measurement study on a real BitTorrent portal, The Pirate Bay (TPB), which is one of the most popular torrent hosting sites. For the purpose of data collection, we leverage the Really Simple Syndication (RSS) feed to detect the availability of a new torrent on TPB and retrieve the torrent information like publisher's username. After downloading the corresponding .torrent file, our monitoring client, which is developed by modifying the Azureus software [4], joins

$\overline{1 \text { http://mmlab.snu.ac.kr/ sbkim }}$ 
the swarm and collects peer lists periodically (once in two hours) from trackers and connected peers in the swarm. For each torrent, the torrent metadata such as its category (e.g., Movie, TV, Porn, etc.) given by the torrent publisher and its published time are recorded. Also the peer information like IP addresses is recorded at our database periodically.

Our datasets, which have been collected for 38 days from March 25 to May 1, 2011 , consist of 51,934 torrents, shared by 16,036,240 users (i.e., IP addresses). We observe that 9,724 TPB accounts (i.e., publishers) have uploaded those 51,934 torrents (i.e., 5.3 torrents per each publisher on average).

\subsection{Publisher Classification}

We classify publishers into three types based on the purposes of content publishing [5]: fake publisher, profit-driven publisher, and altruistic publisher.

1. Fake publishers publish fake contents. They often inject malware into files or make garbage files with catchy titles (e.g., recently released Hollywood movies), often including anti-piracy messages. The administrators of TPB remove their accounts and their associated torrents on TPB when they are reported as fake publishers from users.

2. Profit-driven publishers publish torrents with financial incentives. To promote websites for financial gains, they typically use additional text files including their site URLs, or post the URLs in the textbox on TPB portal. We further divide profit-driven publishers into three types based on what kind of websites are advertised, following [5]: (i) Private BitTorrent trackers who advertise a private BitTorrent portal to tempt people to be its members (They account for $51 \%$ and $67 \%$ of the total number of profit-driven publisher's accounts and the published torrents), (ii) Hosting image websites who promote a number of URLs which are linked to image hosting sites (They account for $26 \%$ and $21 \%$ of the total number of profit-driven publisher's accounts and the published torrents), and (iii) Other websites who advertise their own web sites such as game review sites, adult sites, or comic sharing sites.

3. Altruistic publishers are those who publish their contents without fake contents nor promoting websites.

In order to classify publishers, we take following steps. First, we check whether the publisher's account on TPB is removed or not. If the account is removed and all torrents uploaded by the publisher are not available either, the publisher is concluded as a fake one. We double-check the account of the publisher in the Suprbay forum 2 where users report fake publishers on TPB. When the account is available, we next examine the textbox of each torrent published on TPB. If there are any URLs on the textbox, we identify them as profit-driven publishers.

2 https://forum. suprbay.org 


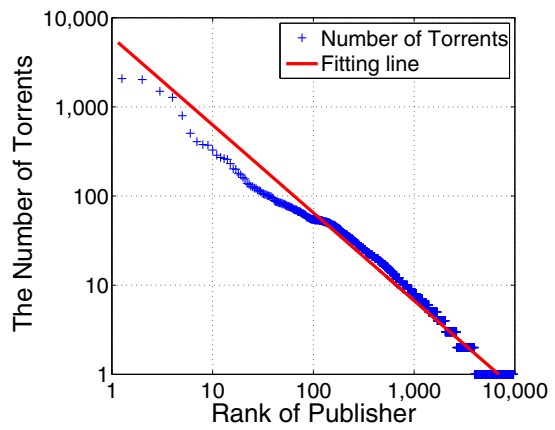

Fig. 1. The number of published torrents by each publisher

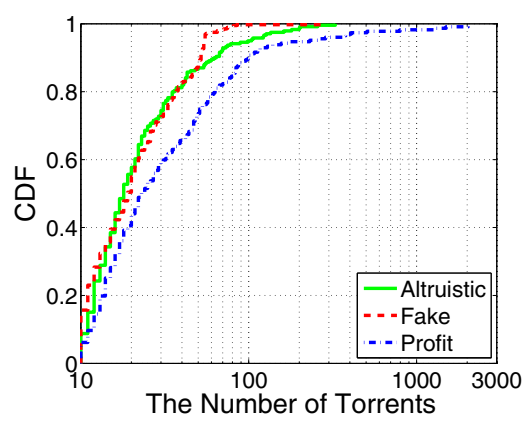

Fig. 2. The number of published torrents by each publisher type

We then examine all the files in our datasets. If there exist additional text files which contain URLs of private BitTorrent trackers or any websites, we classify them as profit-driven publishers. The rest of publishers are classified as altruistic ones. Note that we take into account publishers who have uploaded at least 10 torrents during the data collection period. The number of those publishers considered in this paper is 861 , which accounts for top $9 \%$ of all the publishers and $67 \%$ of the total number of published torrents in our datasets.

\section{The Number of Published Torrents}

In this section, we investigate how many torrents are published by i) each publisher, ii) each publisher type (i.e., altruistic, fake, and profit-driven), and iii) top 100 and the other publishers. The total number of observed publishers in our datasets is 9,724, which have published 51,934 torrents. Among those publishers, we consider 861 publishers who have uploaded at least 10 torrents.

We first plot the number of published torrents by each publisher in Figure 1in decreasing order. Blue plus marks in Figure 1 represent the number of torrents and the red line is the fitting line to those marks. As shown in Figure 1, the curve is straight in log-log scale, which means that a small portion of publishers are responsible for the majority of the torrents, while most of the other publishers each upload a small number of torrents. Among all the publishers, the top 861 publishers are responsible for $67 \%$ of all the published torrents, which are analyzed in the following.

We next examine the number of torrents of each publisher type (i.e., altruistic, fake, and profit-driven). As shown in Figure 2, profit-driven publishers publish more torrents than other publisher types. Note that $10 \%$ of profitdriven publishers publish more than 100 torrents, which explains the heavy tail distribution. This is because profit-driven publishers have financial incentives to upload a large number of torrents [5]. More specifically, top 5 publishers, 


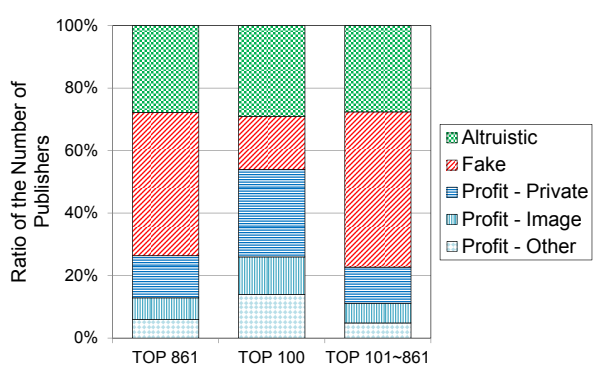

Fig. 3. Ratio of the number of publishers of each publisher type to the total 861 publishers, the top 100, and the next top 101 861 publishers, respectively

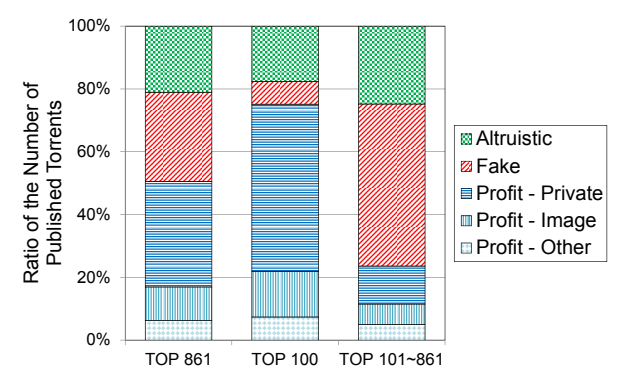

Fig. 4. Ratio of the number of published torrents of each publisher type to the total 861 publishers, the top 100, and the next top 101 861 publishers, respectively

who upload 7,677 torrents (15\% of all the published torrents in our datasets), are profit-driven publishers who promotes private BitTorrent portals. On the contrary, most of fake publishers (98\%) publish less than 60 torrents because their accounts on TPB are frequently removed by the TPB administrator after the reports of fake torrents.

We plot the ratio of the number of publishers and the number of published torrents of each publisher type to the total 861 publishers, the top 100 publishers, and the next top 101 861 publishers in Figures 3 and 4, respectively. Interestingly, $46 \%$ of the 861 publishers are fake ones while the portions of profit-driven and altruistic publishers are $26 \%$ and $28 \%$, respectively, as shown in Figure 3 . Note that profit-driven publishers account for $54 \%$ of publishers among the top 100 publishers, which is in line with the results in [5]. However, around $50 \%$ of the top 101 861 publishers are fake ones, and they are responsible for a significant number of published torrents. Even though fake publishers are banned once their fake content is reported, and hence they cannot upload lots of torrents normally, to be detailed in Section 4, they account for a substantial portion of published torrents since fake publishers are majority of all the publishers in our datasets. We also notice that Figure 4 shows that $51 \%$ of torrents of the 861 publishers are uploaded by profit-driven publishers, and among them, private BitTorrent trackers are responsible for $34 \%$ of the published torrents of the 861 publishers. Among profit-driven publishers, private BitTorrent trackers tend to rank at the top position in terms of the number of published torrents (for instance, top 1 5 profit-driven publishers are private ones), while hosting image websites tend to have lower ranks. This is because private BitTorrent trackers promote BitTorrent portals while hosting image websites just promote private URL links, which will be detailed in Section 6. Since the contribution of the other websites is not so significant, we do not consider them in the following sections. 


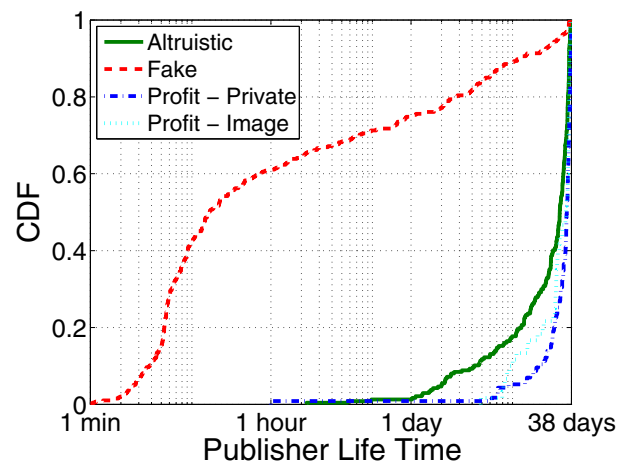

Fig. 5. The estimated lifetime of publishers depending on publisher types

\section{Publisher's Strategies}

BitTorrent publishers uploading contents have their own purposes; altruistic ones wish to share contents, fake ones try to distribute fake contents, and profitdriven ones seek to obtain financial gains [5, 6]. In this section, we analyze their tactical approaches to achieve their own goals. To this end, we seek to answer the following questions: (1) how do they frequently publish contents? (2) which content categories (e.g., tv, movie, and music) are usually selected by each publisher type? and (3) what are the advertising strategies to get financial incentives for profit-driven publishers?

\subsection{Lifetime of Publishers and Their Publishing Rates}

We first estimate the lifetime of a publisher as a time interval between the first content publishing time and the last content publishing time on TPB. As shown in Figure 5, fake publishers have much shorter lifetime than others. Note

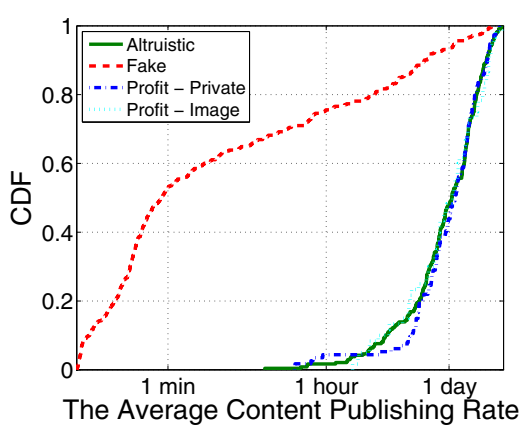

Fig. 6. The average content publishing rate depending on publisher types

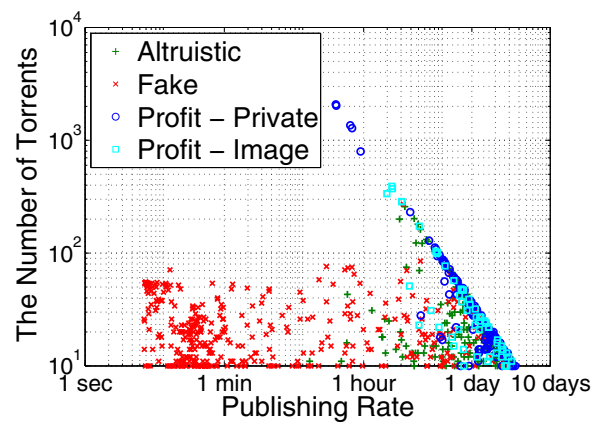

Fig. 7. Publishing rate vs. the number of torrents 


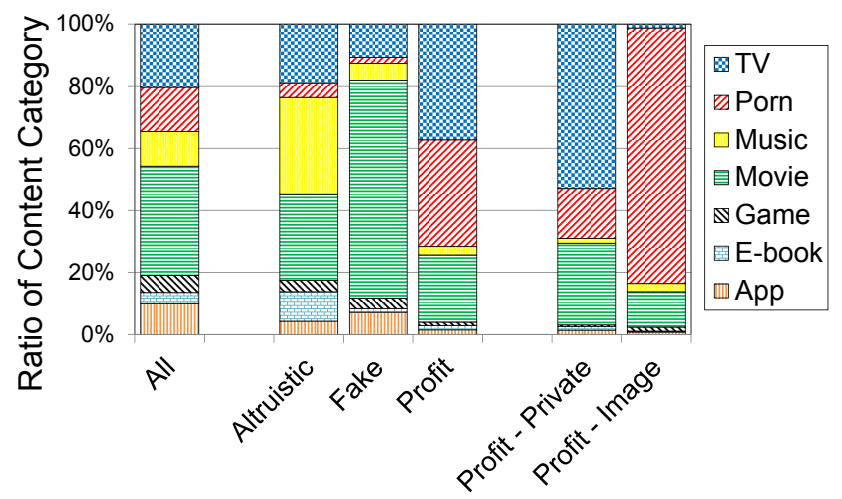

Fig. 8. Ratio of the number of torrents in each category to the total number of torrents depending on publisher types

that about $60 \%$ of fake publishers have less than 1 hour lifetime while most of altruistic and profit-driven publishers have at least 1 day lifetime. The average lifetime of fake publishers is 3.5 days while those of altruistic and profit-driven publishers are 24.6 days and 29.4 days, respectively.

We next examine the publishing rate of each publisher (i.e., the average time interval between two consecutive publications) for each publisher type. As shown in Figure 6, fake publishers publish torrents significantly more frequently than altruistic and profit-driven ones. Fake publishers have high publishing rate (e.g., over $50 \%$ of fake publishers publish contents every one minute or less) because they try to upload as many torrents as possible before their accounts on TPB are banned. Meanwhile, altruistic and profit-driven publishers averagely upload a torrent every single day. However, despite the high publishing rate of fake publishers, the average number of published torrents of each fake publisher (25.1) is not so higher than that of a altruistic/profit-driven publisher (30.8 and 78.0, respectively) as shown in Section 3 because the accounts of fake publishers on TPB are banned shortly.

Figure 7 shows the correlation between publishing rate and the number of published torrents. As shown in Figure 7, except fake publishers, heavy uploaders tend to publish much more frequently than others. Top 5 publishers in terms of the number of published torrents upload contents less than every one hour on average. However, there is no correlation in fake publishers because they try to publish as many torrents as possible until their accounts are removed.

\subsection{Content Categories}

In this subsection, we examine the strategies of publishers depending on the seven major content categories (they account for $92 \%$ and $90 \%$ of the torrent counts and torrent volume, respectively): Movie, Porn, TV, Music, Application, E-book, and Game. 


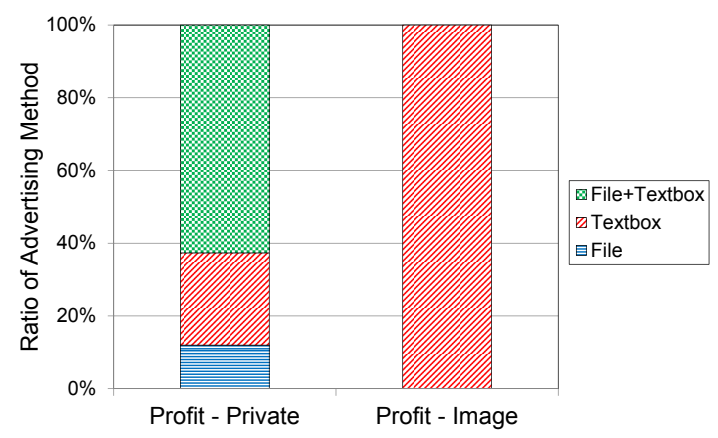

Fig. 9. Ratio of advertising method per profit-driven publisher type

Figure 8 shows the portion of the number of published torrents depending on the content categories for each publisher type. As shown in Figure 8 , around $70 \%$ of torrents published by fake publishers are movie contents because they use the catchy titles such as latest Hollywood movies to attract users. Profit-driven publishers mostly upload TV and Porn contents (more than 70\%). More specifically, private BitTorrent trackers prefer to publish TV contents while hosting image websites prefer to upload porn contents. This is because private BitTorrent trackers often publish popular TV shows and dramas to tempt people to be members of their private BitTorrent portals. Meanwhile, hosting image websites mainly publish porn contents with links to related porn pictures. Clicking the links will lead the users to their websites.

\subsection{Advertising Strategies of Profit-Driven Publishers}

We finally show what strategies are used by profit-driven publishers to advertise their private trackers, or hosting image sites. To this end, we investigate their strategies to promote the URLs in two ways: (i) a name of file (in the torrent) is the URL, and (ii) a textbox (on the web page of TPB) contains the URL. Note that a publisher can adopt multiple strategies. As shown in Figure 9, about $62 \%$ of private BitTorrent trackers use both the file names and textboxes. From any of the two strategies, users can notice the origin of the contents. In contrast, hosting image websites only use textboxes, so that users can click their links even before downloading porn contents. This difference between the adopted strategies of two kinds of profit-driven publishers (i.e., private and image) is due to their revenue models to be discussed in Section 6 .

\section{Downloaders' Behavior}

In this section, we look at the behavior of users (or downloaders), and analyze (1) the number of users who download each torrent, (2) the lifetimes of swarms (i.e., the duration in which there is at least one seed in the swarm), (3) users' sojourn times (i.e., how long each user stays in the swarm), and (4) temporal dynamics 
(in terms of participants) of each swarm, depending on the three publisher types (i.e., altruistic, fake, and profit-driven publishers). Our analysis is further based on the two profit-driven publisher types: private and image.

We first show the average number of downloaders of torrents depending on the publisher types in Figure 10. Note that the central line of each box represents the median, and the top and bottom edges of the boxes indicate the 25th and 75th percentiles, respectively, in Figures 10, 11, and 12. As shown in Figure 10, the number of downloaders of torrents from fake publishers is significantly higher than those of altruistic and profit-driven ones, which indicates that torrents published by fake publishers are in general most popular. The average number of downloaders of a fake publisher's torrent is 32 times and 11 times larger than those of altruistic and profit-driven publisher's ones, respectively. This is because those publishers often publish fake torrents with popular movie titles (e.g., latest Hollywood movies), hence many users are falsely tempted to join the swarms to download. The average number of downloaders of the torrents from profit-driven publishers is 3 times larger than that of the altruistic ones. Note that the 25 th percentile, median, and 75th percentile of the number of downloaders of hosting image websites is 2.4 times, 2.1 times, and 1.4 times larger than those of private BitTorrent trackers as shown in Figure 10, respectively. This is perhaps because the hosting image websites' strategy - uploading popular porn contents, as shown in Section 4.2 - is appealing to the users.

We next show the average swarm lifetime of each torrent depending on the publisher types in Figure 11] The swarm lifetime is the duration in which there is at least one seed in the swarm. As shown in Figure 11, torrents from fake publishers span shorter lifetimes than those of the others, even though there are many more users joining the swarms. Note that the average swarm lifetime of torrents from fake publishers is around 9 days while those of altruistic and profitdriven ones are approximately $17 \sim 18$ days. This is because torrents published by the fake publishers are to be removed from TPB as soon as they are reported. Note that the 25 th percentile, median, and 75 th percentile of the swarm lifetimes of hosting image websites is 3.4 times, 1.6 times, and 1.2 times longer than those of private BitTorrent trackers, respectively. This is because private BitTorrent trackers mainly upload weekly TV shows or dramas which typically attract users

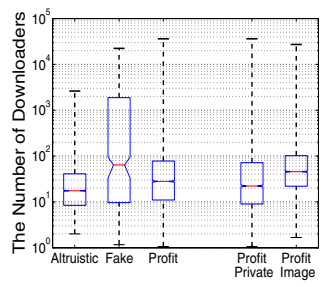

Fig. 10. The number of downloaders depending on publisher types

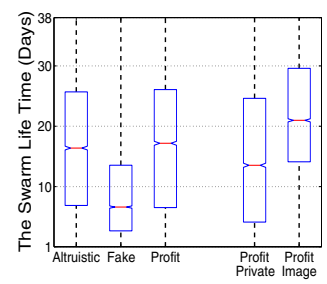

Fig. 11. The swarm lifetime depending on publisher types

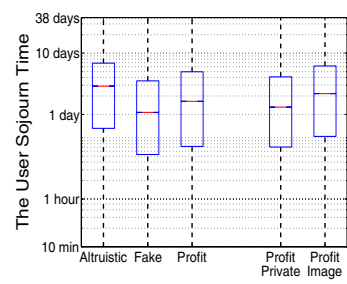

Fig. 12. The user sojourn time depending on publisher types 


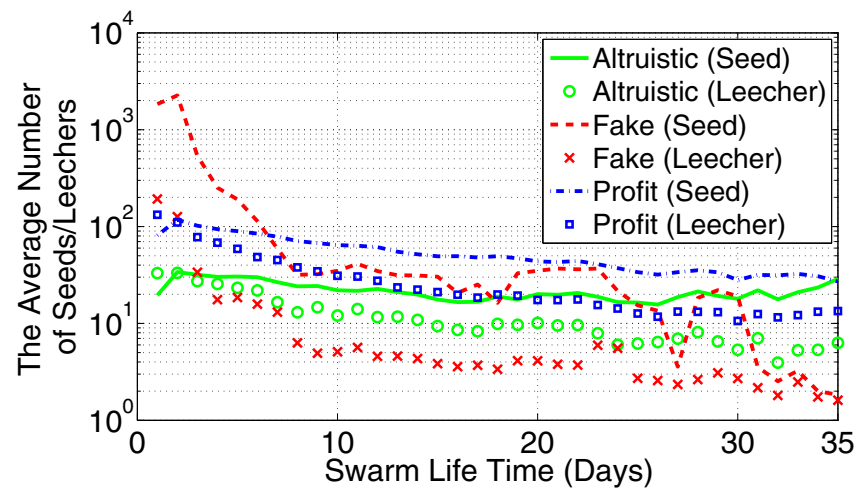

Fig. 13. The average number of seeds/leechers during swarm lifetime

for a shorter period, while hosting image websites mainly upload porn torrents which are attractive relatively long.

To investigate how long users stay in swarms, we show the average sojourn time of users depending on the publisher types in Figure 12. Users of torrents from fake publishers leave the swarms earlier than those of altruistic and profitdriven ones. The average user sojourn time of a fake swarm is 62 hours, while those of altruistic and profit-driven ones are 100 and 84 hours, respectively. Users stay longer in swarms of altruistic publishers. Note that the 25th percentile, median, and 75 th percentile of user sojourn time of hosting image websites is 1.4 times, 1.7 times, and 1.5 times longer than those of private BitTorrent trackers, respectively.

We finally show the average number of seeds and leechers during each day in Figure 13 to examine the temporal dynamics of content popularity in terms of the number of users. There are a significant number of seeds and leechers in the swarms of fake publishers for the first few days, which is in line with Figure 10. However, the number of leechers drops quickly because the torrents published by fake publishes are to be removed from TPB [6]. Torrents published by profit-driven publishers have more seeds and leechers than the ones published by altruistic publishers on average. The average number of seeds and leechers of torrents from profit-driven publishers are 54 and 30 respectively, while those of a torrent of altruistic publishers are 22 and 12 , respectively.

\section{Implications on Publishers' Strategies}

\subsection{Fake Publishers}

From the previous results, we notice that fake publishers are responsible for a large portion of BitTorrent traffic: (1) around 50\% of the publishers who have uploaded at least 10 torrents are fake ones, and they account for $35 \%$ of total published torrents, and (2) the average number of downloaders of a fake 


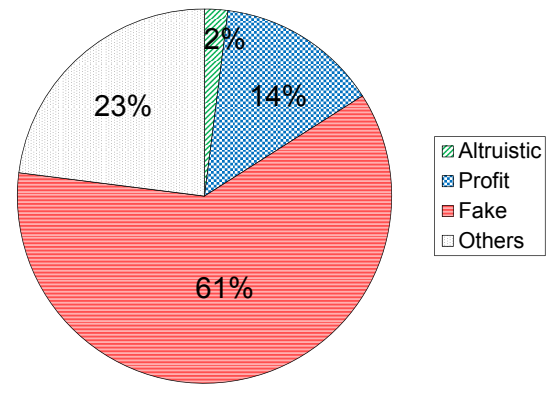

Fig. 14. Ratio of data traffic volume depending on publisher types (Total: 16.1 PB)

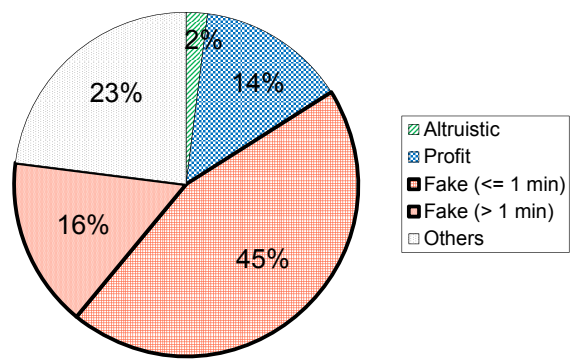

Fig. 15. Ratio of data traffic volume of fake publishers with $<=1$ minute and $>1$ minute publishing rate (Total: $16.1 \mathrm{~PB}$ )

publisher's torrent is 32 times and 11 times larger than those of altruistic and profit-driven publisher's ones, respectively. We estimate the amount of downloading traffic by multiplying the number of published torrents, the size of each torrent, and the number of downloaders of each torren 13 . Figure 14 shows the pie chart of estimated breakdown of downloaded traffic for each type of publisher. Note that "Others" indicates the traffic caused by unclassified publishers, who have uploaded less than 10 torrents according to our datasets. Fake publishers are responsible for around $61 \%$ of the estimated download traffic. If those torrents from fake publishers can be effectively filtered out, a significant amount of BitTorrent-caused traffic will be saved, allowing users to avoid downloading such fake torrents.

To this end, we suggest to use the publishing strategies (or patterns) of fake publishers to effectively detect them. As shown in Section 4.1, fake publishers tactically upload torrents with high publishing rates. In our datasets, $53 \%$ of fake publishers have less than 1 minute publishing rate while none of altruistic and profit-driven publishers do so. Note that $64 \%, 67 \%, 71 \%$, and $76 \%$ of fake publishers have publishing rate less than 5 minutes, 10 minutes, 30 minutes, and 1 hour respectively. In contrast, only $1.7 \%$ and $2.7 \%$ of altruistic and profitdriven publishers have less than 1 hour publishing rate, respectively. Based on this simple publishing patterns of fake publishers, we can automatically detect fake ones in a very efficient manner. For example, if we conservatively filter out publishers who have less than 1 minute publishing rate, around $45 \%$ of download traffic (approximately 7.2 PB) can be saved, as shown in Figure 15 , While the administrators of TPB manually remove fake publisher's accounts and their associated torrents when they are reported as fake ones from other users in the current practice, we believe that this automatic detection will immediately detect the fake publishers, and the potential bandwidth saving will be quite significant.

${ }^{3}$ Note that we do not consider the uploading traffic in this analysis since it is difficult to capture the amount of traffic uploaded by each seed. 


\subsection{Profit-Driven Publishers}

We have divided profit-driven publishers into private BitTorrent trackers and hosting image websites, and analyzed their characteristics. The major difference between the two types of profit-driven publishers (i.e., profit and image) is the revenue model. Private BitTorrent trackers advertise their private BitTorrent portals to attract users to their sites, for possible financial gains through membership fees or donations. To this end, they upload many number of torrents on TPB; top 5 publishers in terms of the number of published torrents are private BitTorrent trackers. Also they use additional text files and the textboxes to advertise their private BitTorrent portals. Whereas, publishers of hosting image websites directly obtain financial rewards from the hosting image websites by redirecting people to click their own URL links. Thus, they only use the textboxes for direct clicks because they can earn financial gains based on the click counts on their URL links. In summary, publishers who want to promote URLs adopt different strategies according to their revenue models. We believe that these publishing strategies can be a reference to the content providers who want to get financial gains in peer-to-peer systems.

\section{Conclusions}

We studied the content publishing and downloading practice on the real BitTorrent system. We investigated the behaviors of 861 (top 9\%) publishers who are responsible for $67 \%$ of all the published torrents on TPB and analyzed the goals of publishers and the strategies of the publishers to achieve their goals. We also observed the user behaviors on TPB to see whether the tactics of the publishers are effective or not. We found that a significant amount of traffic of BitTorrent is generated in downloading fake torrents. Therefore, we suggested a simple but highly effective method to filter out fake publishers on TPB by considering their publishing patterns. We showed that this simple solution can save around $45 \%$ of BitTorrent traffic in our datasets. We also revealed that profit-driven publishers adopt different publishing strategies according to their revenue models. Our ongoing work includes (i) investigation of how the characteristics of publishers affect the performance of BitTorrent swarming systems, and (ii) implementation of the proposed method that detects and filters out fake publishers based on their publishing rate.

Acknowledgment. This work was supported by the IT R\&D program of KCA(11-913-05-002: Fundamental Research on In-network Caching and Routing for Named-data Networking) and the ITRC support program NIPA-2011(C1090-1111-0004) of MKE/NIPA. The ICT at Seoul National University provided research facilities for this study. 


\section{References}

1. Cohen, B.: Incentives Build Robustness in BitTorrent. In: Workshop on Economics of P2P Systems (2003)

2. Instant messaging, one-click hosting and media streaming on the internet, http://www.ipoque.com/en/resources/internet-studies

3. The Pirate Bay, http://thepiratebay.org/

4. Open sourced bittorrent client, Vuze, http://www.vuze.com/

5. Cuevas, R., Kryczka, M., Cuevas, A., Kaune, S., Guerrero, C., Rejaie, R.: Is content publishing in bittorrent altruistic or profit-driven? In: ACM CoNEXT (2010)

6. Kryczka, M., Cuevas, R., Gonzalez, R., Cuevas, A., Azcorra, A.: FakeDetector: A measurement-based tool to get rid out of fake content in your BitTorrent Downloads, arXiv:1105.3671v1 (2011)

7. Zhang, C., Dhungel, P., Wu, D., Ross, K.W.: Unraveling the bittorrent ecosystem. IEEE Transactions on Parallel and Distributed Systems (2010)

8. Le Blond, S., Legout, A., Lefessant, F., Dabbous, W., Ali Kaafar, M.: Spying the world from your laptop. In: USENIX LEET (2010)

9. Legout, A., Liogkas, N., Kohler, E., Zhang, L.: Clustering and sharing incentives in bittorrent systems. In: ACM SIGMETRICS (2007)

10. Legout, A., Urvoy-Keller, G., Michiardi, P.: Rarest first and choke algorithms are enough. In: ACM IMC (2006)

11. Piatek, M., Isdal, T., Anderson, T., Krishnamurthy, A., Venkataramani, A.: Do incentives build robustness in bittorrent. In: USENIX NSDI (2007)

12. Qiu, D., Srikant, R.: Modeling and performance analysis of bittorrent-like peer-topeer networks. In: ACM SIGCOMM (2004) 